\title{
Prevalence of Anemia, Overweight/Obesity, and Undiagnosed Hypertension and Diabetes among Residents of Selected Communities in Ghana
}

\author{
Alex Kojo Anderson \\ Department of Foods and Nutrition, University of Georgia, Athens, GA 30602, USA \\ Correspondence should be addressed to Alex Kojo Anderson; fianko@uga.edu
}

Received 24 February 2017; Accepted 12 July 2017; Published 15 August 2017

Academic Editor: Srinivas Kondalsamy-Chennakesavan

Copyright (C) 2017 Alex Kojo Anderson. This is an open access article distributed under the Creative Commons Attribution License, which permits unrestricted use, distribution, and reproduction in any medium, provided the original work is properly cited.

\begin{abstract}
The increasing numbers of lifestyle related chronic diseases in developing countries call for awareness, early detection, and effective management. The objective of this paper is to report the prevalence of undiagnosed hypertension, diabetes, overweight/obesity, and anemia among residents of selected communities in Ghana. The data comes from a community screening conducted in Ghana as part of the University of Georgia Summer Service Learning Program. Descriptive statistics were used to summarize the data while chi-square and independent $t$-test compared groups. A total of 976 participants ( $37.9 \%$ males and $62.1 \%$ females), 18 years and older, were screened. Mean age was $46.25 \pm 17.14$ years, BMI was $25.44 \pm 5.26 \mathrm{kgm}^{-2}$, and hemoglobin was $12.04 \pm 2.22 \mathrm{~g} / \mathrm{dL} .3 .1 \%$ and $12.6 \%$ reported existing diagnosis for diabetes and hypertension, respectively. Almost half (47.8\%) were overweight/obese; $27.0 \%$ were hypertensive while $34.0 \%$ had diabetes. Also, $28.8 \%$ males compared to $37.8 \%$ females had diabetes $(P=0.015)$, while $28.2 \%$ males compared to $26.2 \%$ females were hypertensive $(P=0.635)$. There were differences in BMI $(P<0.0001)$, anemia $(P=0.007)$, and undiagnosed diabetes $(P<0.0001)$ and hypertension $(P<0.0001)$ by community (Takoradi versus Cape Coast) where the screening took place. Findings from the screening exercise call for improved public health education with a focus on lifestyle habits and health seeking behaviors among Ghanaians.
\end{abstract}

\section{Introduction}

Hypertension (raised blood pressure), one of the major diseases of the 21st century, is reported to affect over 1 billion of the world's population with about three-fourths living in developing countries and almost half (46\%) in Africa [13]. Hypertension is also a major risk factor for a number of chronic diseases including heart disease, heart attack/failure, stroke, cerebrovascular disease, diabetes mellitus, and renal failure. Unfortunately, because of the asymptomatic nature of hypertension during the early stages of the disease, it has not been a priority for primary healthcare workers, particularly in developing countries with limited resources and poor healthcare infrastructure.

Type 2 diabetes (due to insulin insensitivity and/or poor blood glucose management) currently affects 1 in 11 adults, and a predicted 642 million people are expected to be living with diabetes in the world by the year $2040[4,5]$. Recent reports suggest that almost 3 of every 4 diabetics reside in low- and middle-income countries, expected to further increase by the year $2030[6,7]$. Poorly managed diabetes increases the risk of cardiovascular disease, blindness, kidney failure, amputations, and early death. It is projected that the prevalence of diabetes in Africa will increase and surpass those of the other regions of the world by the year 2035 [8-10].

Anemia (low blood hemoglobin concentration) is one of the significant public health problems (hidden hunger) plaguing the world today [11]. Globally, over 1.6 billion people are reported to be anemic [12]. Anemia occurs through the lifecycle but disproportionately affects children and women and individuals from low-income, resource limited areas of the world. Although iron deficiency is the significant cause of anemia, there are other contributing causes of anemia which include parasitic infections, malaria, heavy blood loss as a result of menstruation among females, and other nutrient deficiencies including folate, vitamin $B_{12}$, riboflavin, and copper. Because of the silent nature of anemia, it often goes undiagnosed and poorly managed, leading to poor cognitive 
and physical development of children and negative physical performance and work productivity among adults [13].

The prevalence and risk of death from hypertension and diabetes are on the increase in most developing countries as a result of the ongoing nutrition transition and poor health infrastructure in these countries $[8,14-23]$. The impact of the nutrition transition on the dietary habit is the resulting change from traditionally less processed foods to Western food habits and lifestyles which include the consumption of foods high in calories, sugar, salt, and total fat [24, 25]. These dietary changes have led to increased prevalence of obesity, metabolic syndrome, diabetes, and hypertension in most low- to middle-income countries [26, 27]. This is compounded by poor health seeking behaviors, poor healthcare infrastructure and resource allocation, and inadequate access to preventative health services. Because of the limited healthcare resources, governments of developing countries, including Ghana, tend to focus most of their effort and resources on infectious diseases and not chronic diseases $[19,28]$. Another major problem is the lack of awareness and magnitude of these chronic diseases and their early detection and intervention among populations of developing countries, including Ghana [26]. Thus, in this paper we report the prevalence of undiagnosed hypertension and diabetes, overweight/obesity, and anemia among residents of selected communities in Ghana and their public health implications.

\section{Methods}

2.1. Study Design and Setting. This was a secondary data analysis of data generated from a community nutrition and health screening/education which is part of service learning activities conducted by students from the University of Georgia who participated in a Service Learning Program in Ghana directed by the author. The community nutrition and health screening/education was conducted between June and July 2016. Participants were free living adult community residents who came forward to participate in the screening exercise. The use of the data in this study was approved by the Human Subject Institutional Review Board of the University of Georgia.

The community nutrition and health screening/education exercises occurred in Sekondi-Takoradi, capital city of the Western Region and Cape Coast, capital city of the Central Region of Ghana. The mobile clinics for the nutrition and health screening/education were in two (2) communities (Collins Avenue and Star of the Sea Cathedral) in Takoradi and Apewosika community in Cape Coast.

Sekondi-Takoradi is located at the southeastern part of the Western Region. According to the 2010 Population and Housing Census, the total population of the SekondiTakoradi is 559,548 ( $23.5 \%$ of the region's total population). Over $67 \%$ of the population are 15 years and older. Over $96 \%$ of the population in Sekondi-Takoradi resides in urban areas. Almost $91 \%$ of the population in the Metropolis are literate (having some form of formal education) while $90 \%$ of the adult population are employed. Health facilities include the regional hospital, 2 district hospitals, and a number of clinics that provide healthcare services to residents of the area and other neighboring communities.

The total population of Cape Coast is 169,894 (constituting about $7.7 \%$ of the total population of the Central Region) of which over 120,000 are 15 years and older. Cape Coast is predominantly urban with three-quarters of the population residing in urban areas. Over $90 \%$ of the population of Cape Coast is literate compared to $78.2 \%$ and $74.1 \%$ for the Central Region and Ghana, respectively. Almost $91 \%$ of adults are employed. Cape Coast is the home of the regional hospital which also serves as a referral center. It also has a district hospital and a number of clinics that provide healthcare to the population of the Metropolis.

2.2. Data Collection Procedures. Data for this study was generated from adults 18 years and older who voluntarily participated in the University of Georgia Ghana Summer Service Learning from June to July 2016. The data available included community where the screening occurred, age, gender, weight, height, blood hemoglobin, fasting blood glucose, and blood pressure.

2.3. Anthropometric Measurement. Weight and height were measured using calibrated weighing scale and stadiometer following standard protocol [29]. Because these anthropometric measurements were conducted in the open, the requirement for minimum clothing during weight measurement was not possible. Weight was measured with participants wearing their regular clothing devoid of other clothing accessories in the case of women and wallets and so forth in the pocket of men. Weight was measured to the nearest $0.1 \mathrm{~kg}$. Height was measured to the nearest $0.1 \mathrm{~cm}$ without shoes, cap, or headgear. Weight and height measurements were used to estimate body mass index (BMI = weight in $\mathrm{kg} \div[\text { height in meters }]^{2}$ ). BMI was further classified as underweight $\left(\mathrm{BMI}<18.5 \mathrm{kgm}^{-2}\right)$, normal weight $(\mathrm{BMI}=18.5-$ $\left.24.9 \mathrm{kgm}^{-2}\right)$, overweight $\left(\mathrm{BMI}=25.0-29.9 \mathrm{kgm}^{-2}\right)$, and obese $\left(\mathrm{BMI} \geq 30.0 \mathrm{kgm}^{-2}\right)$.

2.4. Hemoglobin. Blood hemoglobin level was measured using HemoCue $\mathrm{Hb}_{201}{ }^{+}$. To measure blood hemoglobin level, the patient's forefinger was prepared by wiping the tip with alcohol to sterilize the area and then allowed to dry. The sterilized area was then pricked with a lancet and a drop of blood collected into a microcuvette. The microcuvette filled with the blood was then inserted into the HemoCue Hb201 for the hemoglobin measurement. The measured hemoglobin was classified as normal $(\mathrm{Hb}>13 \mathrm{~g} / \mathrm{dL}$ for men and $\mathrm{Hb}>$ $12 \mathrm{~g} / \mathrm{dL}$ for women), mild anemia (Hb: 11.0-12.9 g/dL for men versus $\mathrm{Hb}$ : $11.0-11.9 \mathrm{~g} / \mathrm{dL}$ for women), moderate anemia ( $\mathrm{Hb}$ : $8.0-10.9 \mathrm{~g} / \mathrm{dL})$, and severe anemia $(\mathrm{Hb}<8 \mathrm{~g} / \mathrm{dL})$.

2.5. Fasting Blood Glucose. Blood glucose concentration was measured using HemoCue Glucose $201^{+}$. For blood glucose measurement, the patient forefinger was prepared as described for hemoglobin measurement above. A drop of blood was collected from a finger prick into a microcuvette and inserted into the HemoCue Glucose $201^{+}$for blood glucose measurement. The measured blood glucose was later 
classified as normal (FBG < $100 \mathrm{mg} / \mathrm{dL})$, prediabetes (FBG: $100-125 \mathrm{mg} / \mathrm{dL}$ ), and diabetes (FBG $>126 \mathrm{mg} / \mathrm{dL}$ ) [30].

2.6. Blood Pressure. Blood pressure was measured at least 10 minutes from the patient's arrival at the mobile screening clinic. Participants were seated and made comfortable with one arm resting on a table. The BP was measured using an automated (digital) sphygmomanometer. The cuff was inflated by turning on the sphygmomanometer for the blood pressure measurement. The measured blood pressure was classified as normal (systolic: less than $120 \mathrm{mmHg}$; diastolic: less than $80 \mathrm{mmHg}$ ), prehypertension (systolic: 120-139 mmHg; diastolic: $80-90 \mathrm{mmHg}$ ), and hypertension (systolic: $\geq 140 \mathrm{mmHg}$; diastolic $\geq 90 \mathrm{mmHg}$ ).

2.7. Statistical Analysis. The statistical package IBM SPSS Statistics 23 for Windows (IBM SPSS Inc., Armonk, NY, USA) was used for data entry and analysis. Descriptive statistics were used to summarize the data and results reported as percentages (frequencies) and means ( \pm standard deviation). Student's unpaired, two-sided $t$-test was used to compare means between Takoradi and Cape Coast. Pearson Chisquared test (Fisher's exact test) was used to compare the independent variables (place of resident, age, and gender) and outcome variables (indicators of anemia, obesity, diabetes, and hypertension). All $P$ values were two-sided, and criterion for statistical significance was set at $P<0.05$.

\section{Results}

A total of 976 adults (597 from Takoradi and 379 from Cape Coast) participated in the screening with data for the study. The average age was $46.25 \pm 17.14$ (range: 18-100) years and was similar in the two Metropolises. Majority of the participants were over 40 years old. Participants were fairly distributed by gender $(P=0.437)$ in the two cities with over $60 \%$ being females (Table 1 ). For individuals who participated in the screening exercise, $12.6 \%$ (13.7\% in Takoradi versus $10.8 \%$ in Cape Coast) and $3.1 \%$ (3.7\% in Takoradi versus $2.1 \%$ in Cape Coast) reported known (existing) diagnosis for hypertension and diabetes, respectively.

3.1. Participant Hemoglobin. The mean hemoglobin level was $12.04 \pm 2.22 \mathrm{~g} / \mathrm{dL}$ (range: $2.00-17.80$ ). There were differences in hemoglobin levels by gender and screening community. The mean blood hemoglobin concentration was significantly lower among females compared to males $(11.43 \pm 2.17 \mathrm{~g} / \mathrm{dL}$ versus $13.03 \pm 2.17 \mathrm{~g} / \mathrm{dL}, P<0.0001)$ and Takoradi compared to Cape Coast $(11.85 \pm 2.38 \mathrm{~g} / \mathrm{dL}$ versus $12.34 \pm 1.92 \mathrm{~g} / \mathrm{dL}$, $P=0.001)$, although these were within the normal range. Overall, $47.0 \%$ of the participant had mild to severe anemia. About a third of males (30.5\%) and a little over half (52.2\%) of females had mild to severe anemia according to their blood hemoglobin concentration (Figure 1). Prevalence of anemia was significantly $(P=0.007)$ higher among participants from Takoradi compared to their counterparts from Cape Coast (Table 1).

3.2. Participant Weight. Overall, almost half (47.8\%) of the participants were either overweight or obese $\left(\mathrm{BMI}>25 \mathrm{kgm}^{-2}\right)$.
The average BMI was $26.06 \pm 5.32 \mathrm{kgm}^{-2}$ versus $24.45 \pm$ $5.01 \mathrm{kgm}^{-2}$ for participants in Takoradi and Cape Coast, respectively $(P<0.0001) .18 .4 \%$ of the participants were obese. Whereas $53.7 \%$ of participants from Takoradi were either overweight or obese, only $38.4 \%$ of those from Cape Coast were overweight or obese $(P<0.0001)$. The prevalence of overweight/obesity was higher among female participants compared to male participants $(P<0.0001)$ (Figure 1).

3.3. Blood Pressure. Generally, measured systolic and diastolic blood pressures were higher for participants from Takoradi compared to those from Cape Coast. The measured blood pressure shows that majority of the participants were either prehypertensive or hypertensive. The overall prevalence of hypertension was $27.0 \%$. Of those with hypertensive blood pressure, only $12.6 \%$ (123/976) had prior diagnosis for hypertension. The prevalence of hypertension detected from the screening exercise was significantly $(P<0.0001)$ higher among participants from Takoradi (30.4\%) compared to their counterparts from Cape Coast $(21.8 \%)$. There was overall significant $(P=0.002)$ difference in hypertension by participant gender, with males having slightly higher prevalence compared to females (Figure 1). Similar trends were observed in Takoradi and Cape Coast (data not shown).

3.4. Fasting Blood Glucose. Majority of the participants had fasting blood sugar levels classified as either prediabetes or diabetes. The measured fasting blood glucose showed $39.2 \%$ prevalence for diabetes of which 3.1\% (30/976) reported known diabetes diagnosis. Almost $40 \%$ of participants from Takoradi were diabetics compared to $24.6 \%$ from Cape Coast based on their measured fasting blood sugar $(P<0.0001)$ (Table 1). Most (39.8\%) of the participants from Cape Coast compared to $28.4 \%$ from Takoradi had measured fasting blood sugar level in the normal range. There was statistical difference in fasting blood sugar level by participant gender with $37.1 \%$ of females compared to $28.8 \%$ of males who were diabetics $(P=0.015)$ (Figure 1$)$.

\section{Discussion}

Ghana, as is the case of many low- and middle-income countries, is dealing with the triple burden of malnutrition (undernutrition, micronutrient deficiencies, and increasing prevalence of overweight/obesity and related chronic diseases) because of changes in their food systems due to their degree of economic development and adopting Western food culture [31]. The situation poses a threat to the healthcare infrastructure and productivity and creates a financial burden for these countries. There is therefore a need to bring attention to awareness and early intervention to mitigate the human resource and economic burden of these countries. In this study, we examined the problem of undiagnosed hypertension and diabetes, overweight/obesity, and anemia among residents of Takoradi and Cape Coast, Ghana. We observed high prevalence of undiagnosed hypertension and diabetes, overweight/obesity, and anemia among residents who participated in the screening exercise. 
TABLE 1: Characteristics of participants.

\begin{tabular}{|c|c|c|c|}
\hline & $\begin{array}{c}\text { Takoradi } \\
\text { Mean } \pm \text { SD }\end{array}$ & $\begin{array}{l}\text { Cape Coast } \\
\text { Mean } \pm \text { SD }\end{array}$ & $P$ value \\
\hline Age (years) & $46.45 \pm 16.69$ & $45.92 \pm 17.86$ & 0.637 \\
\hline BMI $\left(\mathrm{kgm}^{-2}\right)$ & $26.06 \pm 5.32$ & $24.45 \pm 5.01$ & $<0.0001$ \\
\hline Systolic blood pressure (mmHg) & $136.89 \pm 24.80$ & $134.27 \pm 25.53$ & 0.114 \\
\hline Diastolic blood pressure ( $\mathrm{mmHg}$ ) & $86.98 \pm 14.62$ & $81.81 \pm 14.04$ & $<0.0001$ \\
\hline Fasting blood glucose (mg/dL) & $123.46 \pm 43.41$ & $112.86 \pm 38.37$ & $<0.0001$ \\
\hline \multirow[t]{2}{*}{ Hemoglobin $(\mathrm{g} / \mathrm{dL})$} & $11.85 \pm 2.38$ & $12.34 \pm 1.92$ & 0.001 \\
\hline & $n(\%)$ & $n(\%)$ & \\
\hline \multicolumn{4}{|l|}{ Age (years) } \\
\hline$<25$ & $61(10.2)$ & $45(11.9)$ & \multirow{4}{*}{0.661} \\
\hline $25-40$ & $177(29.6)$ & $120(31.7)$ & \\
\hline $41-60$ & $222(37.2)$ & $136(35.9)$ & \\
\hline$>60$ & $137(22.9)$ & $78(20.6)$ & \\
\hline \multicolumn{4}{|l|}{ Sex } \\
\hline Male & $228(38.2)$ & $142(37.5)$ & \multirow{2}{*}{0.437} \\
\hline Female & $369(61.8)$ & $237(62.5)$ & \\
\hline \multicolumn{4}{|l|}{ Weight status } \\
\hline Underweight & $21(3.5)$ & $25(6.6)$ & \multirow{4}{*}{$<0.0001$} \\
\hline Normal weight & $255(42.8)$ & $208(55.0)$ & \\
\hline Overweight & $196(32.9)$ & $90(23.8)$ & \\
\hline Obese & $124(20.8)$ & $55(14.6)$ & \\
\hline \multicolumn{4}{|l|}{ Measured blood pressure } \\
\hline Normal & $204(34.2)$ & $184(48.5)$ & \multirow{3}{*}{$<0.0001$} \\
\hline Prehypertensive & $211(35.4)$ & $113(29.8)$ & \\
\hline Hypertensive & $181(30.4)$ & $82(21.6)$ & \\
\hline \multicolumn{4}{|l|}{ Fasting blood glucose } \\
\hline Normal & $169(28.4)$ & $149(39.8)$ & \multirow{3}{*}{$<0.0001$} \\
\hline Prediabetes & $189(31.8)$ & $133(35.6)$ & \\
\hline Diabetes & $237(39.8)$ & $92(24.6)$ & \\
\hline \multicolumn{4}{|l|}{ Hemoglobin } \\
\hline Normal & $305(51.1)$ & $211(56.1)$ & \multirow{4}{*}{0.007} \\
\hline Mild anemia & $150(25.1)$ & $100(26.6)$ & \\
\hline Moderate anemia & $98(16.4)$ & $56(14.9)$ & \\
\hline Severe anemia & $44(7.4)$ & $9(2.4)$ & \\
\hline
\end{tabular}

The findings of the present study show overall prevalence of undiagnosed hypertension and diabetes to be 53.2\% (77.3\% in Takoradi and $0.0 \%$ in Cape Coast) and $90.9 \%(90.5 \%$ in Takoradi and $91.3 \%$ in Cape Coast), respectively. Our finding is consistent with findings from a study by Amoah and colleagues conducted in Accra, Ghana, where they reported prevalence of undiagnosed diabetes [32]. However, the prevalence of undiagnosed diabetes among participants in the current study from Takoradi and Cape Coast is higher than reported by Amoah and colleagues [32]. This suggests a lack of awareness or knowledge of symptoms and general education of these diseases among residents of Takoradi and Cape Coast. This is a major public health problem as previous studies have shown that awareness and early detection leads to effective management of hypertension and diabetes [3336]. This observation paints a bleak health picture for both Takoradi and Cape Coast as this stands to affect the work productivity and quality of life of the adult population of these areas, which will eventually have a negative impact on their economic growth as these adults constitute the workforce. This situation also has the potential of posing a great challenge to the healthcare infrastructure and increasing healthcare costs for the government of Ghana and the individuals involved as well as their respective families.

The prevalence of overweight and obesity was disproportionately higher among participants from Takoradi compared to those from Cape Coast $(P<0.0001)$. Over half of participants from Takoradi compared to about a third from Cape Coast were either overweight or obese. This is higher than previously reported among the adult population in Ghana and other developing countries [37]. For example, a recent systematic review and analysis conducted by OforiAsenso and colleagues of 43 studies conducted across the 10 regions of Ghana reported that about $43 \%$ of the adult 


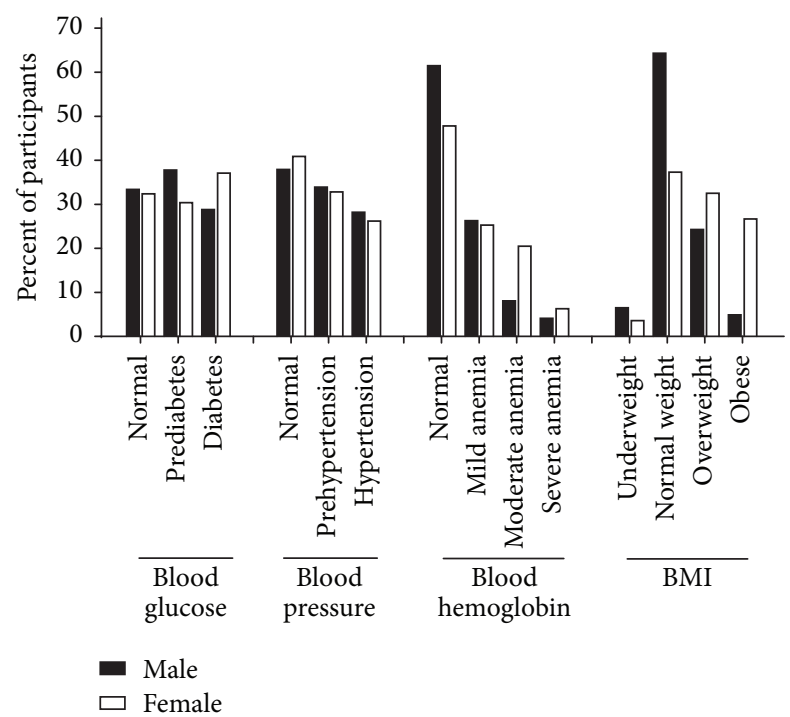

Figure 1: Prevalence of undiagnosed diabetes and hypertension, anemia, and obesity.

population are either overweight or obese. Women generally had a higher prevalence of overweight/obesity compared to men [37]. This differential and high prevalence rate of overweight/obesity by gender as was observed in the current study has been attributed to cultural perception of body size. In most African countries including Ghana, being overweight or obese is associated with good health, wellbeing, and high social class [20, 23, 38-43]. Prevailing knowledge of the strong association between obesity and chronic diseases including hypertension and diabetes paints a gloomy picture of the health of Ghanaian adults should this continue [4446]. There is therefore a need for culturally appropriate interventions that target healthy weight management among Ghanaian adults, especially women, so they understand the health risks associated with overweight and obesity and that being overweight or obese is not necessarily an indication of good health status and wellbeing.

The prevalence of anemia observed among participants in this study was similar to that reported in a number of systematic reviews of global level data [11, 12, 47]. This observation is a significant public health problem as anemia has direct effect on one's performance and productivity. In the current study we could not ascertain the cause of the high anemia prevalence due to our inability to obtain information on potential causes and general lifestyle of the participants. The high anemia prevalence could be due to a number of factors including genetic make-up (such as sickle cell trait and thalassemia), dietary habits (micronutrient deficiencies), infections such as hookworm and schistosomiasis, and disease states such as malaria or inflammation [48]. Other evidences suggest increased risk for anemia among patients with diabetes [49]. This is evidential in the current study with high prevalence of undiagnosed diabetes and anemia.

This study comes with a number of limitations which should inform the interpretation of the findings. First, this is a secondary data analysis, and the data used came from a community screening exercise, thus the likelihood of selection bias. Second, we did not have data to examine the predictors of anemia, overweight/obesity, hypertension, and diabetes. Third, the screening exercise took place within communities in Takoradi and Cape Coast; therefore, our findings may not be representative of respective regions limiting the generalizability of the findings; thus results should be interpreted with caution. However, the study had a large sample size, which is a strength, and also highlights the lack of awareness of hypertension, diabetes, overweight/obesity, and anemia in communities in Ghana to inform public health interventions.

\section{Conclusions}

The situation of high prevalence of obesity, anemia, and undiagnosed hypertension and diabetes has significant public health implications for health planners of a country such as Ghana which has limited health infrastructure with a heavy burden of undernutrition and infectious diseases among its population. There is therefore an urgent need for health policies focused on primary prevention and early detection of these chronic disease conditions within the Ghanaian population. The ongoing nutrition and epidemiologic transition and urbanization in Ghana have affected the traditional food culture and general lifestyle. The situation poses a serious threat to the wellbeing, quality of life, and productivity of Ghana's workforce if immediate steps are not taken to reverse these rising trends.

\section{Ethical Approval}

The proposal for this paper was reviewed and approved by the Human Subjects Institutional Review Board of the University of Georgia.

\section{Disclosure}

Because the study only used data generated from a community health and nutrition screening exercise, waiver was given for documenting participant consent.

\section{Conflicts of Interest}

The author declares no conflicts of interest.

\section{Authors' Contributions}

Alex Kojo Anderson conceived the idea, sought IRB approval, conducted the data analysis, and wrote the manuscript.

\section{Acknowledgments}

First and foremost, the author would like to thank all University of Georgia students who participated in the 2016 Ghana Summer Service Learning Program who worked diligently to screen residents of selected communities in Ghana. The author also wants to thank Evan S. Johnson for entering and cleaning the data for analysis and, lastly, the residents of Takoradi and Cape Coast in Ghana who participated in the screening exercise that generated the data for this paper. 


\section{References}

[1] WHO, The world health report 2002: reducing risks, promoting healthy life, World Health Organization, Geneva, Switzerland, 2002.

[2] WHO, WHO Global Report. Preventing chronic disease a vital investment, World Health Organization, Geneva, Switzerland, 2005.

[3] WHO, A global brief on HYPERTENSION. Silent killer, global public health crisis, World Health Organization, Geneva, Switzerland, 2013.

[4] International Diabetes Federation (IDF), Primary Care Diabetes Europe (PCDE), and World Heart Federation (WHF), The Berlin Declaration: a Collective Ambition for Policy Change to Drive Early Action in Type 2 Diabetes, International Diabetes Federation, Berlin, Germany, 2016.

[5] IDF Diabetes Atlas, International Diabetes Federation (IDF), 2015.

[6] R. Sicree, J. Shaw, and P. Zimme, "Diabetes and Impaired Glucose Tolerance," in proceedings of the International Diabetes Federation, Geneva, Switzerland, 2010.

[7] C. Murray and A. D. Lopez, Global Burden of Disease: A Comprehensive Assessment of Mortality and Disability from Diseases, Injuries and Risk Factors in 1990 and Projected to 2020, Harvard School of Public Health, Cambridge, MA, USA, 1996.

[8] L. Guariguata, D. Whiting, I. Hambleton, J. Beagley, U. Linnenkamp, and J. Shaw, "Global estimates of diabetes prevalence for 2013 and projections for 2035," Diabetes Research and Clinical Practice, vol. 103, no. 2, pp. 137-149, 2014.

[9] J. E. Shaw, R. A. Sicree, and P. Z. Zimmet, "Global estimates of the prevalence of diabetes for 2010 and 2030," Diabetes Research and Clinical Practice, vol. 87, no. 1, pp. 4-14, 2010.

[10] J. Mattei, V. Malik, N. M. Wedick et al., "Reducing the global burden of type 2 diabetes by improving the quality of staple foods: The Global Nutrition and Epidemiologic Transition Initiative," Globalization and Health, vol. 11, no. 1, article no. 23, 2015.

[11] E. McLean, M. Cogswell, I. Egli, D. Wojdyla, and B. De Benoist, "Worldwide prevalence of anaemia, WHO Vitamin and Mineral Nutrition Information System, 1993-2005," Public Health Nutrition, vol. 12, no. 4, pp. 444-454, 2009.

[12] N. J. Kassebaum et al., "A systematic analysis of global anemia burden from 1990 ti 2010," Blood, vol. 23, pp. 615-624, 2014.

[13] WHO, "Iron deficiency anaemia: assessment, prevention, and control," in A guide for Programme Managers, World Health Organization, Geneva, Switherland, 2001.

[14] P. M. Kearney, M. Whelton, K. Reynolds, P. Muntner, P. K. Whelton, and J. He, "Global burden of hypertension: analysis of worldwide data," The Lancet, vol. 365, no. 9455, pp. 217-223, 2005.

[15] R. Funetes et al., "Hypertension in developing economies: a review of population-based studies carried out from 1980-1998," Journal of Hypertension, vol. 18, pp. 521-529, 2000.

[16] R. Edwards, N. Unwin, F. Mugusi et al., "Hypertension prevalence and care in an urban and rural area of Tanzania," Journal of Hypertension, vol. 18, no. 2, pp. 145-152, 2000.

[17] J. Tran, M. Mirzaei, and S. Leeder, "Hypertension: its prevalence and population-attributable fraction for mortality from stroke in the Middle East and North Africa," Circulation, vol. 122, article e155, 2010.

[18] D. Yach, C. Hawkes, C. L. Gould, and K. J. Hofman, "The global burden of chronic diseases: overcoming impediments to prevention and control," The Journal of the American Medical Association, vol. 291, no. 21, pp. 2616-2622, 2004.

[19] V. Perkovic, R. Huxley, Y. Wu, D. Prabhakaran, and S. MacMahon, "The burden of blood pressure-related disease: a neglected priority for global health," Hypertension, vol. 50, no. 6, pp. 991997, 2007.

[20] A. M. Prentice, "The emerging epidemic of obesity in developing countries," International Journal of Epidemiology, vol. 35, no. 1, pp. 93-99, 2006.

[21] WHO, "Global Strategy on Diet, Physical Activity \&amp; Health," in Obesity and Overweight, World Health Organization, Geneva, Switherland, 2003.

[22] B. M. Popkin and P. Gordon-Larsen, "The nutrition transition: Worldwide obesity dynamics and their determinants," International Journal of Obesity, vol. 28, pp. S2-S9, 2004.

[23] L. McLaren, "Socioeconomic status and obesity," Epidemiologic Reviews, vol. 29, no. 1, pp. 29-48, 2007.

[24] J. Schmidhuber and P. Shetty, "The nutrition transition to 2030: why developing countries are likely to bear the major burden," in Proceedings of the 97th Seminar of European Association of Agricultural Economists, vol. 2, pp. 150-166, Food and Agriculture Organization (FAO), Reading, UK, 2005.

[25] B. M. Popkin, L. S. Adair, and S. W. Ng, "Global nutrition transition and the pandemic of obesity in developing countries," Nutrition Reviews, vol. 70, no. 1, pp. 3-21, 2012.

[26] M. M. Ibrahim and A. Damasceno, "Hypertension in developing countries," The Lancet, vol. 380, no. 9841, pp. 611-619, 2012.

[27] G. Danaei, M. M. Finucane, J. K. Lin et al., "National, regional, and global trends in systolic blood pressure since 1980: systematic analysis of health examination surveys and epidemiological studies with 786 country-years and 5.4 million participants," The Lancet, vol. 377, no. 9765, pp. 568-577, 2011.

[28] O. Adeyi, O. Smith, and S. Robles, Public Policy and the Challenge of Chronic Noncommunicable Diseases, World Bank, Washington, DC, USA, 2007.

[29] R. S. Gibson, Principles of Nutritional Assessment, Oxford University Press, Oxford, UK, 2005.

[30] Centers for Disease Control and Prevention, "Current trends CDC criteria for anemia in children and childbearing-aged women," Morbidity and Mortaligy Weekly Report (MMWR), vol. 38, no. 22, pp. 400-404, 1989.

[31] B. M. Popkin, "Global nutrition dynamics: the world is shifting rapidly toward a diet linked with noncommunicable diseases," The American Journal of Clinical Nutrition, vol. 84, no. 2, pp. 289-298, 2006.

[32] A. G. Amoah, S. K. Owusu, and S. Adjei, "Diabetes in Ghana: a community based prevalence study in Greater Accra," Diabetes Research and Clinical Practice, vol. 56, no. 3, pp. 197-205, 2002.

[33] M. M. Ibrahim, H. Rizk, L. J. Appel et al., "Hypertension prevalence, awareness, treatment, and control in Egypt: Results from the Egyptian National Hypertension Project (NHP)," Hypertension, vol. 26, no. 6 I, pp. 886-890, 1995.

[34] C. Agyemang, M. A. Bruijnzeels, and E. Owusu-Dabo, "Factors associated with hypertension awareness, treatment, and control in Ghana, West Africa," Journal of Human Hypertension, vol. 20, no. 1, pp. 67-71, 2006.

[35] K. Wolf-Maier, R. S. Cooper, H. Kramer et al., "Hypertension treatment and control in five european countries, Canada, and the United States," Hypertension, vol. 43, no. 1, pp. 10-17, 2004.

[36] P. Primatesta and N. R. Poulter, "Improvement in hypertension management in England: results from the Health Survey for 
England 2003," Journal of Hypertension, vol. 24, no. 6, pp. 11871192, 2006.

[37] R. Ofori-Asenso, A. A. Agyeman, A. Laar, and D. Boateng, "Overweight and obesity epidemic in Ghana - A systematic review and meta-analysis," BMC Public Health, vol. 16, no. 1, article no. 1239, 2016.

[38] P. Bovet, A. G. Ross, J.-P. Gervasoni et al., "Distribution of blood pressure, body mass index and smoking habits in the urban population of Dar es Salaam, Tanzania, and associations with socioeconomic status," International Journal of Epidemiology, vol. 31, no. 1, pp. 240-247, 2002.

[39] P. Craig, V. Halavatau, E. Comino, and I. Caterson, "Perception of body size in the Tongan community: Differences from and similarities to an Australian sample," International Journal of Obesity, vol. 23, no. 12, pp. 1288-1294, 1999.

[40] N. J. Pollock, "Cultural elaborations of obesity-fattening practices in pacific societies," Asia Pacific Journal of Clinical Nutrition, vol. 4, pp. 357-360, 1995.

[41] M. Holdsworth, A. Gartner, E. Landais, B. Maire, and F. Delpeuch, "Perceptions of healthy and desirable body size in urban Senegalese women," International Journal of Obesity, vol. 28, no. 12, pp. 1561-1568, 2004.

[42] T. Puoane, J. M. Fourie, M. Shapiro, L. Rosling, N. C. Tshaka, and A. Oelofse, "Big is beautiful" - An exploration with urban black community health workers in a South African township," South African Journal of Clinical Nutrition, vol. 18, no. 1, pp. 615, 2005.

[43] M. Rguibi and R. Belahsen, "Body size preferences and sociocultural influences on attitudes towards obesity among Moroccan Sahraoui women," Body Image, vol. 3, no. 4, pp. 395-400, 2006.

[44] G. A. Colditz, W. C. Willett, A. Rotnitzky, and J. E. Manson, "Weight gain as a risk factor for clinical diabetes mellitus in women," Annals of Internal Medicine, vol. 122, no. 7, pp. 481-486, 1995.

[45] E. S. Ford, D. F. Williamson, and S. Liu, "Weight change and diabetes incidence: findings from a national cohort of US adults," American Journal of Epidemiology, vol. 146, no. 3, pp. 214-222, 1997.

[46] R. Scranton, H. D. Sesso, M. J. Stampfer, J. W. Levenson, J. E. Buring, and J. M. Gaziano, "Predictors of 14-year changes in the total cholesterol to high-density lipoprotein cholesterol ratio in men," American Heart Journal, vol. 147, no. 6, pp. 1033-1038, 2004.

[47] G. A. Stevens, M. M. Finucane, L. M. De-Regil et al., "Global, regional, and national trends in haemoglobin concentration and prevalence of total and severe anaemia in children and pregnant and non-pregnant women for 1995-2011: a systematic analysis of population-representative data," The Lancet Global Health, vol. 1, no. 1, pp. E16-E25, 2013.

[48] World Health Organization, Assessing the iron status of populations, World Health Organization, Geneva, Switherland, 2007.

[49] D. R. Bosman, A. S. Winkler, J. T. Marsden, I. C. Macdougall, and P. J. Watkins, "Anemia with erythropoietin deficiency occurs early in diabetic nephropathy," Diabetes Care, vol. 24, no. 3, pp. 495-499, 2001. 


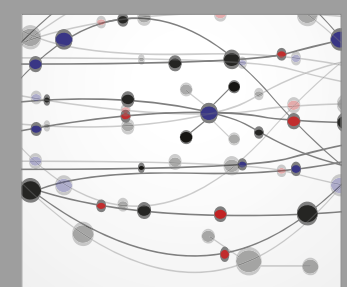

The Scientific World Journal
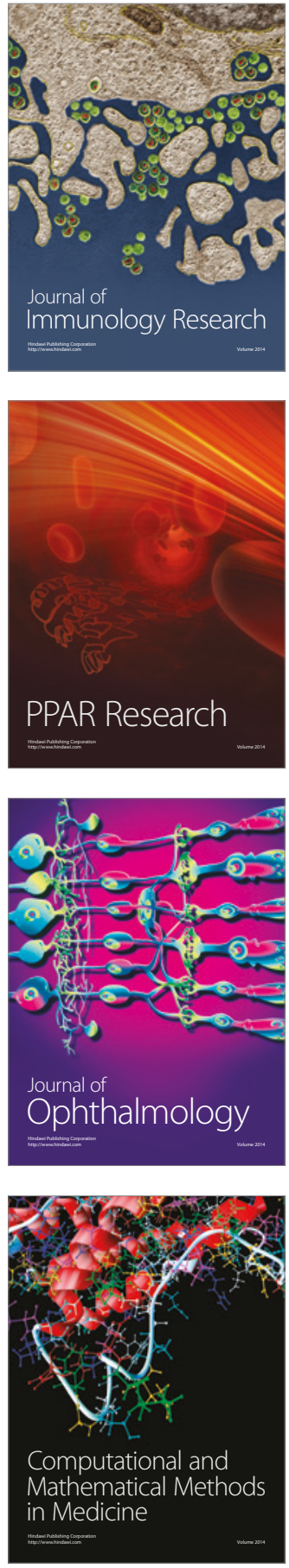

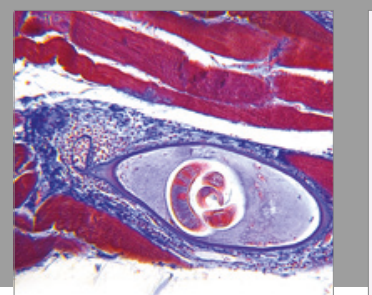

Gastroenterology Research and Practice
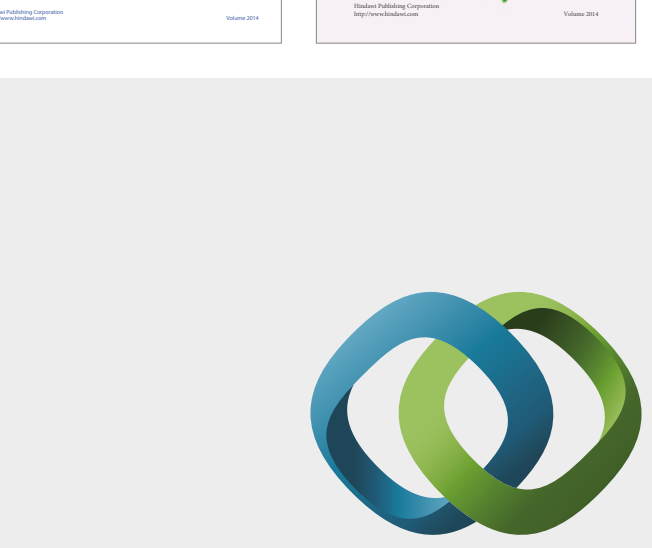

\section{Hindawi}

Submit your manuscripts at

https://www.hindawi.com
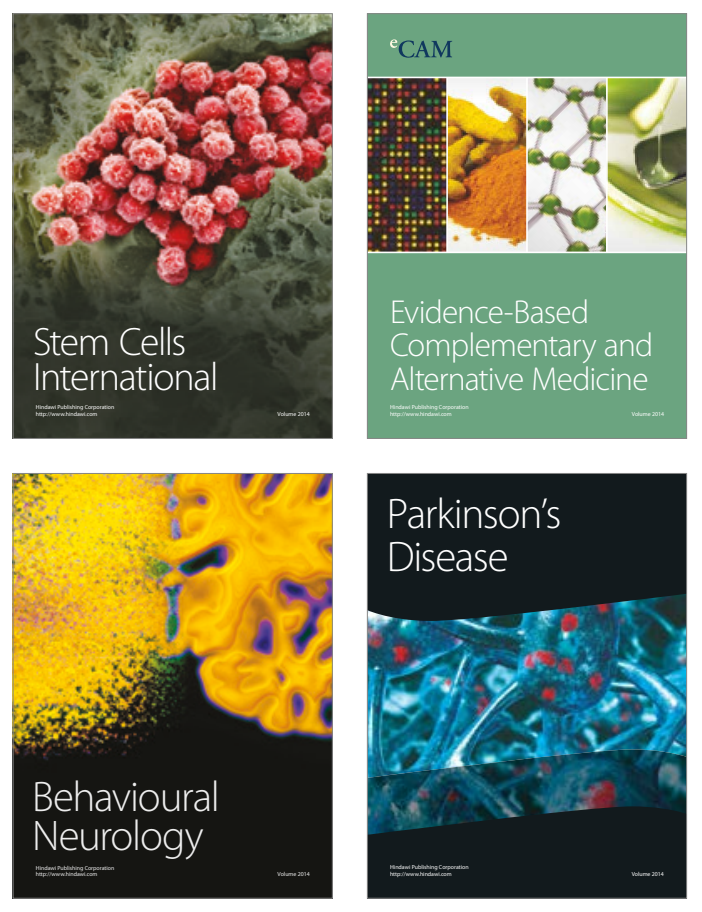
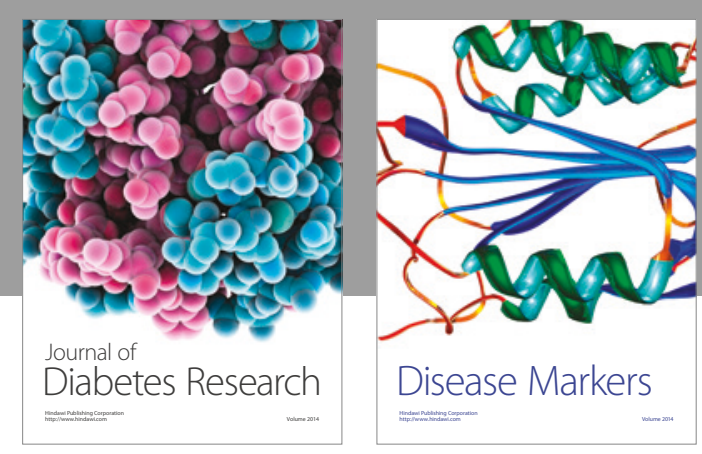

Disease Markers
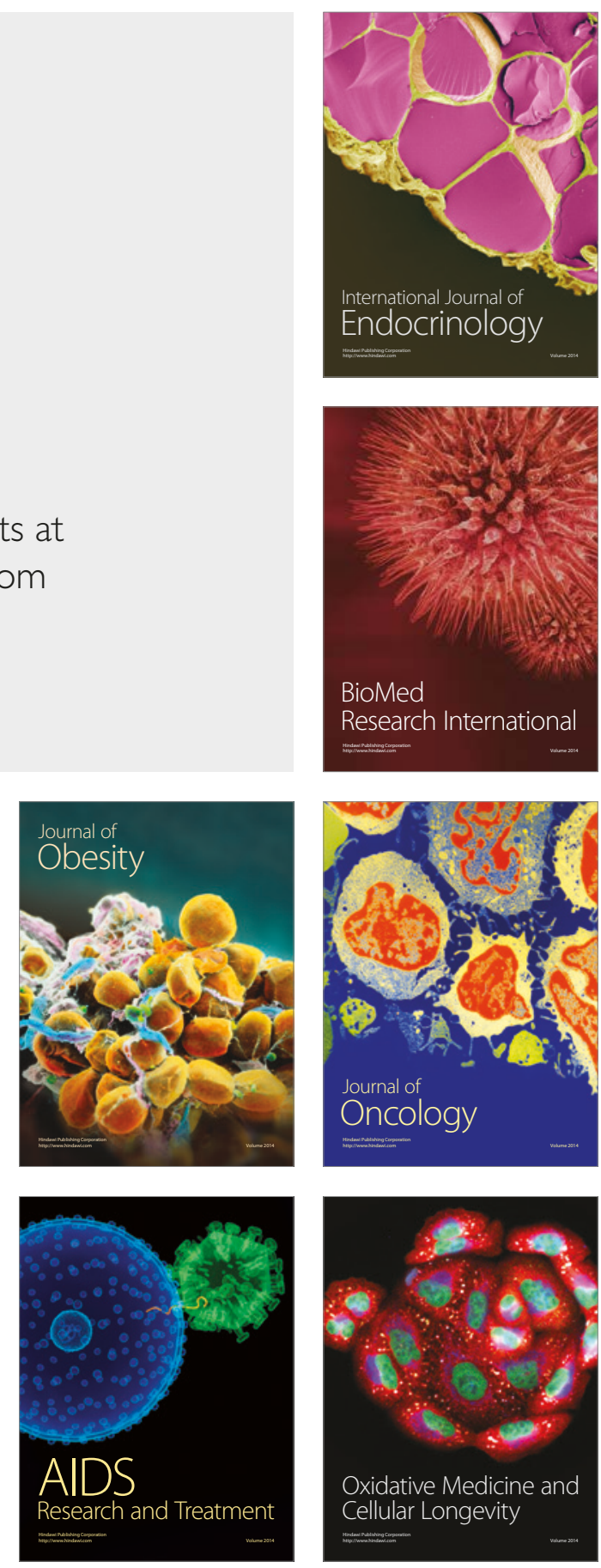